\section{Stephanie Blum}

\section{Milchsäurebakterien und Gesundheit}

\section{Das Anwendungsspektrum von Probiotika bei Erwachsenen}

Die Zunahme ernährungswissenschaftlicher Erkenntnisse hat dazu geführt, dass der Zusammenhang zwischen Fehlernährung und dem Auftreten bestimmter chronischer Erkrankungen heute besser verstanden wird. Zusätzlich steigt der Kenntnisstand, wie solche Erkrankungen durch Ernährung (spezifische Nahrungsmittel als Teil der Gesamternährung) positiv beeinflusst werden können. Lebensmittel, die bestimmte probiotische Milchsäurebakterien enthalten, sind ein Beispiel dafür, wie Nahrungsmittel zusätzliche gesundheitsfördernde Effekte ausüben können. Ein gut untersuchter Effekt spezifischer probiotischer Bakterienstämme ist deren Einfluss auf das Immunsystem. Die in vielen Studien nachgewiesene Stärkung der Immunabwehr ist im Hinblick auf die moderne Lebensweise, vielfach geprägt durch Stress, unregelmässige Nahrungsaufnahme oder häufige Einnahme von Antibiotika, ein nicht vernachlässigbarer Nutzen. Darüber hinaus wurde in verschiedenen Studien die klinische Relevanz für definierte Milchsäurebakterien gezeigt. Dies gilt insbesondere für gastrointestinale Störungen, wie Diarrhöen unterschiedlicher Ätiologie, oder die Verbesserung der Helicobacter pylori-assoziierten chronischen Magenschleimhautentzündung. Obwohl die bisherigen Studien zeigen, dass der Verzehr probiotischer Milchsäurebakterien ebenso unbedenklich ist wie der von konventionellen (Joghurt-) Kulturen, muss die Sicherheit jedes probiotischen Bakteriums a priori gezeigt werden. In diesem Zusammenhang sind wissenschaftliche Studien zur Beschreibung der molekularen Mechanismen, die dem gezeigten gesundheitsfördernden Effekt zugrunde liegen, erforderlich. Zusammen mit der Analyse des bakteriellen Genoms sind dies Schritte zu einer optimierten Nutzen-RisikoAbschätzung. Sind die genannten Anforderungen erfüllt, können in naher Zukunft vermehrt ausreichend charakterisierte probiotische Stämme zur Unterstützung der Therapie in spezifischen klinischen Anwendungsbereichen sinnvoll eingesetzt werden.

Schlüsselwörter: P robiotika, Darmflora, Immunabwehr, Gastroenteriden, Helicobacter pylori, chronisch entzündliche Darmerkrankungen

\section{Probiotic bacteria and health}

The use of probiotics in adults

The scientific literature demonstrates that probiotic bacteria may provide a wide range of health-beneficial effects going beyond basic nutrition. One of the frequently described benefits associated with the consumption of probiotics in foods is the stimulation of the immune system resulting in improved body resistance. To date the immune system is increasingly compromised by an unbalanced lifestyle, stress or frequent intake of antibiotics. Different human studies have demonstrated the clinical relevance of specific probiotic microorganisms, especially in the management of gastrointestinal disorders, such as diarrhea of various etiologies or in the improvement of Helicobacter pylori-associated chronic gastritis. Although the safety of probiotic bacteria has been demonstrated by a variety of studies, proper risk assessment should be performed for every (new) probiotic microorganism which is intended for human consumption. In this context, the studies on the molecular mechanisms of probiotic health associated effects and the analysis of the bacterial genome are valid tools for future benefit-risk assessments. By this, in the near future, the use of selected probiotic strains as nutritional support of current therapies can be extended to clinical applications.

Keywords: Probiotics, intestinal flora, immune defense, chronic gastrointestinal disorders, Helicobacter pylori, chronic gastritis 
aber auch gefriergetrocknet als Nahrungsergänzungsprodukt (Supplement) dargeboten werden. In allen Fällen handelt es sich um die Verabreichung von lebenden Bakterien, deren Wirksamkeit im Produkt durch wissenschaftliche Verfahren nachgewiesen werden muss.

Gemäss der Definition sind Probiotika lebende Mikroorganismen, die nach ihrem Verzehr in adäquater Anzahl gesundheitsfördernde Effekte auf Mensch und Tier ausüben, welche über den grundlegenden ernährungsphysiologischen Nutzen des damit verzehrten Nahrungsmittels hinausgehen [1]. In der Regel handelt es sich hierbei um spezifische Stämme probiotisch aktiver Lactobazillen und Bifidobakterien. Besonders robuste Probiotika zeigen eine hohe Magen-Darm-Resistenz und adhärieren an das Darmepithel. Dies erhöht ihre Verweildauer im Magen-Darm-Trakt, ohne das Gleichgewicht der endogenen Mikroflora langfristig zu beeinträchtigen. Vielfach wurde gezeigt, dass dies zur Verbesserung der intestinalen Barriere-Funktion beiträgt und zusätzlich die Kolonisierung des Darms durch enteropathogene Keime reduzieren kann [2] (Abb. 1).

Seit einiger Zeit wird diskutiert, ob die klassische Definition der lebenden Probiotika auf abgetötete Mikroorganismen oder bakterielle Komponenten (z.B. Zellwandbestandteile) erweitert werden sollte. Dies erfordert jedoch weitere experimentelle und klinische Studien zur Wirksamkeit.

\section{Mikroflora, Immunsystem und Gesundheit}

Der menschliche Organismus ist permanent potentiell pathogenen Keimen ausgesetzt. Ein gut funktionierendes Immunsystem wird in den meisten Fällen Infektionen verhindern oder ausheilen können. Störungen der Immunabwehr werden jedoch immer häufiger. Faktoren wie Stress, unregelmässige oder Fehlernährung, steigender Alkoholgenuss oder die unkritische Einnahme von Antibiotika tragen zur allgemeinen Schwächung des Immunsystems bei. Auch der Alterungsprozess führt zu einer verminderten

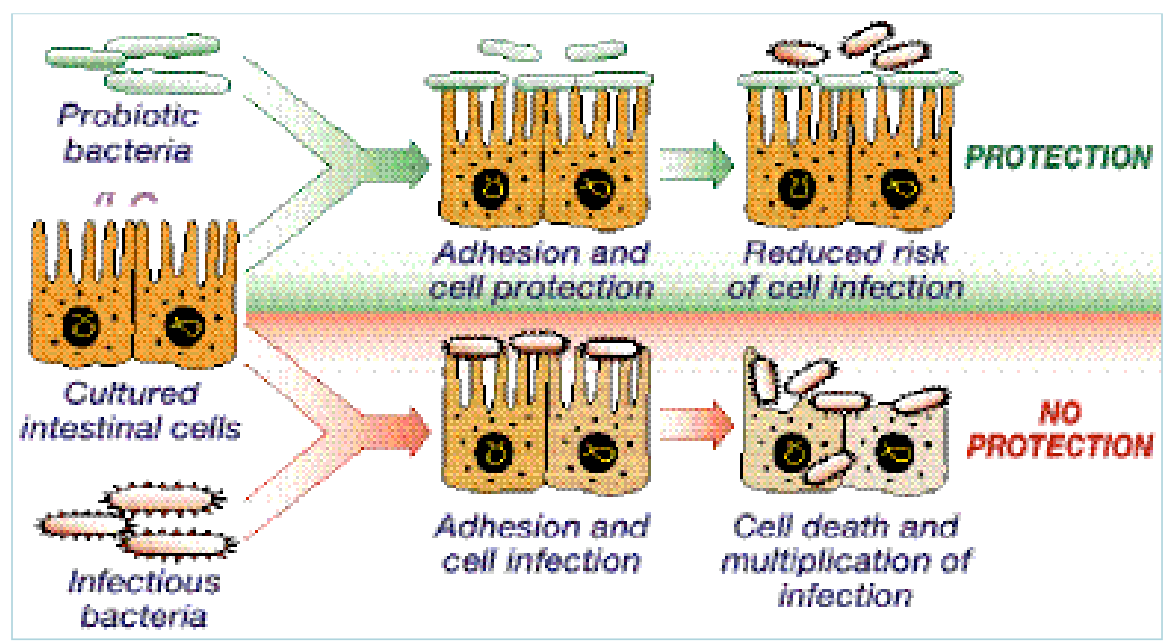

Abb. 1. Kompetitive Verdrängung von Enteropathogenen im Darm durch Probiotika.

Leistungsfähigkeit des Immunsystems.

Die Mikroflora selbst spielt eine grosse Rolle bei lokalen intestinalen Abwehrmechanismen.

Präventive und therapeutische Effekte, wie etwa Verkürzung von Durchfallerkrankungen oder verringerte Inzidenz, wurden für verschiedene probiotische Bakterien, darunter Bifidobacterium lactis Bb12 und Lactobacillus rhamnosus GG, gezeigt [(3,4);Saavedra, 2000; 777 /id; Saavedra, 1994; 1047 /id].

\section{Stärkung der unspezifischen Immunabwehr durch Probiotika}

Neben der angeborenen (innate) unspezifischen Immunabwehr entwickelt sich im Verlauf des Lebens in Kontakt mit der Vielzahl von Antigenen die erworbene spezifische Immunität.

In den letzten Jahren ist die Bedeutung der unspezifischen Immunabwehr u.a. durch die Identifizierung von Rezeptoren, die Strukturen an Bakterien erkennen und eine Immunantwort vermitteln, in den Vordergrund gerückt. Die unspezifische Immunabwehr zählt daher zu den elementaren Abwehrmechanismen unseres Körpers, die eingedrungene Keime aufspüren und schnell beseitigen. Eine zentrale Rolle spielen hierbei Phagozyten (Monozyten, Granulozyten) und natürliche Killer-Zellen (NK) im Blut.

Eine der ersten Humanstudien mit
L. johnsonii NCC533 [5] zeigte, dass bei regelmässigem Verzehr des probiotischen Mikroorganismus die unspezifische Phagozytose-Aktivität im Blut von gesunden Probanden erhöht wurde. Die Stimulierung der Phagozyten war Dosis-abhängig [109 Bakterien/Tag) und der Effekt war auch einige Wochen nach Ende der Einnahme nachweisbar [6]. Eine parallele Analyse der Mikroflora zeigte die transiente Kolonisierung der Probanden mit L. johnsonii NCC533, jedoch keine Veränderung der allgemeinen Mikroflora-Zusammensetzung.

Im Zusammenhang eines europäischen Projektes (Probdemo CT-961028) wurden diese Beobachtungen bestätigt und darüber hinaus gezeigt, dass die Immunstimulierung durch $L$. johnsonii La1 ohne entzündliche Prozesse ablief (keine Erhöhung der Entzündungsmarker CRP, Albumin, Oromucosoid oder IL-6). Eine weitere, interessante Beobachtung resultierte aus dieser Studie. Probanden, die Träger des opportunistischen Bakteriums Clostridium perfringens waren, zeigten nach Verzehr von L. johnsonii La1 deutlich niedrigere Keimzahlen dieses Bakteriums im Darm auf. Daraus wurde geschlossn, dass L. johnsonii La1 zur gesunden Mikroflora-Darm Homeostase beiträgt [7].

Das Immunsystem des älteren Menschen wird durch L. paracasei NCC2461 positiv beeinflusst. In Kombination mit Vitaminen und Folsäure konnte, im Vergleich zu der unsupplementierten 
Kontrollgrupe, eine Erhöhung der natürlichen Killerzell-(NK)-Aktivität und eine geringere Inzidenz von Infektionen gezeigt werden $[8,9]$.

\section{Stärkung der spezifischen Immunantwort}

Der am besten charakterisierte $\mathrm{Ab}$ wehrmechanismus in der Darmmukosa ist die Produktion von sekretorischem Immunglobulin A (sIgA) gegen Toxine, Bakterien oder Viren. Antigenspezifische Immunglobuline vom Typ A (IgA) werden von B-Zellen in der Mukosa produziert und gekoppelt an die sekretorische Kette in das Darmlumen sezerniert. Die durch die Bindung an IgA neutralisierten Antigene werden sodann eliminiert. Dieser Prozess läuft permanent und ohne Aktivierung des Komplementsystems ab.

Die Effizienz der IgA-Produktion kann durch Probiotika gesteigert werden, wie einige Studien gezeigt haben. L. johnsonii La1 (1010cfu/Tag) erhöhte die spezifische IgA-Antwort nach Impfung mit einem attenuierten Salmonellen-Impfstoff (Typh21) [10]. In einer weiteren klinischen Studie erhöhte $L$. johnsonii La1 die Gesamtzahl an IgAAntikörpern im Darmlumen und im Blut [11].

\section{Probiotika in der Therapie von Gastroenteriden und Diarrhöen}

Akute Gastroenteritis, verbunden mit Diarrhöen, ist eine häufig auftretende intestinale Störung, die sowohl bei Kindern als auch bei Erwachsenen zu beobachten ist. Obgleich die Ätiologien unterschiedlich sind, handelt es sich um Störungen des natürlichen Gleichgewichts der Mikroflora durch bakterielle/virale Infektionen oder als Folge einer Antibiotikabehandlung. In der Regel werden Durchfallerkrankungen durch orale Rehydratisierung behandelt, insbesondere bei Kindern und älteren Menschen. Obgleich unerlässlich, führt dies jedoch zu keiner Verkürzung der Durchfalldauer.

Die Behandlung mit Antibiotika hat eine Abnahme der sogenannten Kolo-

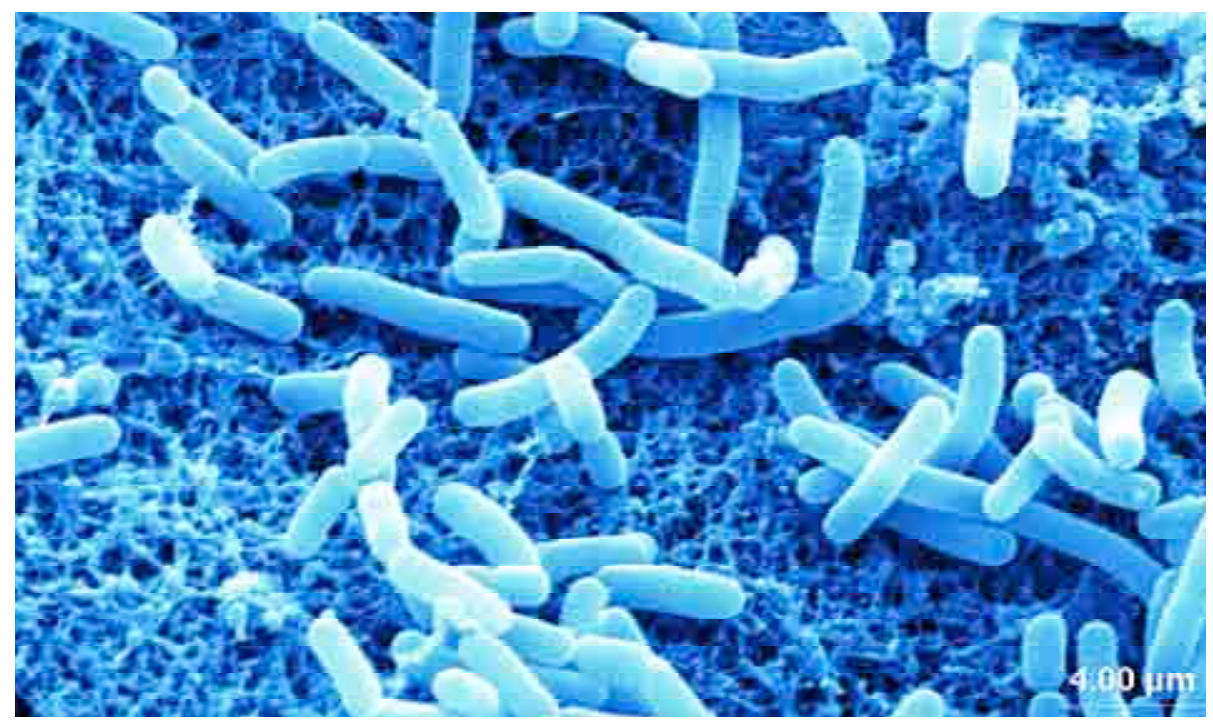

Abb. 2. Lactobacillus johnsonii La1.

nisationsresistenz und metabolischen Aktivität der Mikroflora zur Folge, die zur Antibiotika-assoziierten Diarrhöe führt. Randomisierte, Placebo-kontrollierte klinische Untersuchungen haben gezeigt, dass spezifische probiotische Bakterien die Antibiotika-assoziierte Diarrhöe verhindern oder verkürzen können. Klinische Evidenz wurde u.a. für die probiotische Hefe Saccharomyces boulardii, Lactobacillus rhamnosus GG und Enterococcus faecium SF68 gezeigt $[12,13]$.

Auch bei Gastroenteriden, die durc $h$ pathogene Bakterien vom Typ Clostridium difficile,oder Vi ren (Rotavirus bei Kindern) verursacht werden, zeigten L. rhamnosus GG $[4,14]$ und Bifidobacterium lactis Bb12 in klinischen Studien präventive und partiell kurative Effekte [15]. Eine Meta-Analyse unterschiedlicher Studiendaten konnte zeigen, dass die Verabreichung von Probiotika das Risiko einer Durchfallepisode von mehr als 3 Tagen Dauer signifikant reduziert hatte [16].

Experimentelle Studien legen nahe, dass durch den gezielten Einsatz von spezifischen probiotischen Mikroorganismen die Ansiedlung von pathogenen Keimen im Darm durch die Bildung von Bakterioziden, Milchsäure und sogenannten Biosurfactants verhindert wird. Gleichzeitig trägt wohl auch die Aktivierung der mukosalen Immunabwehr zur effizienten Prävention oder Heilung bei.

Die Kosten-Effizienz des Einsatzes von spezifischen probiotischen Mikroorganismen zur Behandlung von Durchfallerkrankungen ist ein wesentlicher Aspekt, gerade im Hinblick auf den vermehrten Einsatz von Antibiotika. Experten empfehlen schon heute den Einsatz von Probiotika in der Prävention der Clostridium difficile-bedingten Gasteoenteritis, insbesondere bei Risikogruppen, wie etwa älteren Menschen oder Personen, die rezidive Durchfallerkrankungen hatten.

\section{Heliobacter pylori Infektionen}

Heliobacter-pyloriInfektionen treten weltweit auf. Laut Statistiken sind etwa $50 \%$ der Weltbevölkerung mit $H$. pylori infiziert. Viele infizierte Personen entwickeln im Laufe der Zeit eine chronische Gastritis, die asymptomatisch verlaufen kann. Die Infizierung mit H. pylori erfolgt meistens im Kindesalter und kann spontan abheilen. H. pylori-positive Erwachsene bleiben jedoch lebenslang infiziert. $H$. pylori ist auch eine häufige Ursache bei der Entstehung von Ulcera im Magen.

In drei klinischen Studien in $\mathrm{Zu}$ sammenarbeit mit der Gastroenterologischen Abteilung der Universitätsklinik Lausanne (CHUV) wurde der Einfluss von L. johnsonii NCC533 (LC1 Sauermilchprodukt, $10^{10}$ Bakterien/ Tag) oder L. johnsonii NCC533 Kulturüberstand auf die Heliocobacter py- 
lori-assoziierte Gastritis getestet [17].

In allen drei Studien, die entweder mit a) L. johnsonii NCC533 Kulturüberstand (+/- Omeprazol), b) LC1 (+ Clarithomyxcin) oder c) LC1 allein (in asymptomatischen $H$. pylori-infizierten Patienten) arbeiteten, konnte die Verringerung der Infektion mit $H$. pylori dokumentiert werden. In den beiden letzten Studien, in denen lebende Bakterien (in Form von LC1) verabreicht wurden, konnte darüber hinaus eine Verbesserung der Gastritis durch Reduktion der Entzündungsreaktion beobachtet werden. Dies wiederum zeigt deutlich die immuno-modulatorischen Eigenschaften von $L$. johnsonii NCC533 [18,19].

Aufgrund der anti-entzündlichen Wirkung von L. johnsonii NCC533 können Produkte, die das probiotische Bakterium enthalten, als Teil der täglichen Ernährung dazu beitragen, die H. pylori-assoziierte Magenentzündung einzuschränken.

\section{Chronisch entzündliche Darmerkrankungen}

Chronisch entzündliche Darmerkrankungen, wie Morbus Crohn oder Colitis ulcerosa, entwickeln sich mit zunehmender Häufigkeit. Obgleich die Ätiologie unklar ist, gelten folgende $\mathrm{Zu}$ sammenhänge als kausal: eine genetische Disposition (z.B. eine Mutation im NOD2-Gen, etwa in 30\% aller CrohnPatienten), die enterische Mikroflora und eine Dysfunktion des mukosalen Immunsystems. Zahlreiche präklinische und klinische Studien zeigten, dass eine inadäquate, unkontrollierte Immunantwort gegen kommensale Bakterien der autologen Mikroflora die chronisch entzündlichen Prozesse in der Darmmukosa initiieren und propagieren. Das bedeutet, dass die orale Toleranz gegen die Kommensalen im Darm nicht mehr gewährleistet ist.

Neue Forschungsansätze ziehen auch probiotische Konzepte (aufgrund der immuno-modulatorischen Aktivität einiger Stämme) als diätetische Ergänzung zu den verschiedenen Standardtherapien hinzu.

Erste klinische Studien führten positive Effekte auf. So konnte die
Einnahme von VLS3 (lyophilisierte Lactobazillen und Bifidobakterien) $\mathrm{zu}$ einer Verbesserung der Rückfallrate in Patienten mit Pouchitis führen [20].

Jedoch zeigten sich nicht alle verwendeten Stämme als erfolgversprechend. Eine klinische Studie an Crohn'sPatienten, in der die Einnahme des Probiotikums L. rhamnosus GG dazu beitragen sollte, die Patienten länger in Remission $\mathrm{zu}$ halten (Rückfallrate beim M. Crohn ca. 50\%), zeigte keine positiven Effekte des Bakteriums im Vergleich zur Placebogruppe [21].

Diese und ähnliche Studien zeigen das Potential von spezifischen, nicht allen, probiotischen Bakterien im gegebenen klinischen Kontext. Dabei sind die Eigenschaften des Probiotikums und des medizinischen «Targets» von erheblicher Bedeutung, um erfolgreich - mit ernährungsphysiologischen Mitteln - die traditionellen und biologischen Therapien zu unterstützen. Derartige Studien liefern auch immer Daten für eine sichere Anwendung des spezifischen probiotischen Mikroorganismus in der klinischen Situation.

\section{Charakterisierung probiotischer \\ Mikroorganismen}

Obgleich die Effizienz von Probiotika beim Menschen im Vordergrund steht, gibt es auch eine Vielzahl von Unters uchungen zur mikrobiologischen Charakterisierung der probiotischen Stämme. Seit kurzem sind auch - als Weiterentwicklung der molekularen Möglichkeiten - gesamte Genomanalysen von probiotischen Bakterien publiziert, so die Genomsequenz von L. johnsonii NCC533 [22] und Bifidobacterium longum NCC2705 [23]. Diese Daten liefern wichtige Informationen zur Sicherheit der Stämme, wie etwa Antibiotika-Resistenzgene, potentielle Pathogenizitäts- und Virulenzfaktoren oder integrierte Plasmide. Darüber hinaus wird versucht, von den bakteriellen Genen (oder deren Expression im Tiermodell) auf probiotische Effekte zu schliessen, um so genannte probiotische Merkmale oder eine molekulare Handschrift des betreffenden Stammes (molecular signature) zu erstellen. Ist dies erfolgt, lassen sich spezifische Sonden (PCR, polymerase chain reaction oder FISH, fluorescence in situ hybridisation) herstellen, die es in der Zukunft erlauben, neue Stämme sehr viel schneller und zielgerichtet auf potentielle probiotische Funktionalität zu testen.

\section{Präklinische Untersuchungen}

Eine Vielzahl von experimentellen Invitro- und In-vivo-Studien wurden im Zusammenhang mit probiotischen Stämmen publiziert. In vitro wurde vor allem die direkte oder indirekte Interaktion von probiotischen Mikroorganismen mit Zellen des Darmepithels [24,25] oder Immunzellen wie Makrophagen [5,26], Leukozyten [27,28] oder NK Zellen [29] untersucht.

In der Regel lassen sich stammspezifische Induktion von Zytokinen und Chemokinen an Zielzellen beobachten, die Aktivitäts- und Effizienzsteigerung von Phagozyten oder Zunahme der unspezifischen Killeraktivität in NK-Zellen nachweisen.

Auch anhand dieser Untersuchungen wird deutlich, dass die durch Probiotika induzierten Effekte an menschlichen oder tierischen Zellen stammspezifisch sind, was sich auch später in humanen Effizienzstudien zeigt. Ein wichtiger Aspekt ist ebenfalls die Dosis des probiotischen Mikroorganismus. Basierend auf zahlreichen Untersuchungen lässt sich heute verbindlich sagen, das zwischen $10^{6}$ und $10^{8}$ Bakterien im Darm vorhanden sein müssen, um probiotische Effekte messen zu können (siehe auch Kommentar des Bundesinstitut für gesundheitlichen Verbraucherschutz und Veterinärmedizin (BGVV), Deutschland). Da während der Magen-Darmpassage mit einem Verlust von ca. 1-2 log zu rechnen ist (die Überlebensrate ist ebenfalls stammspezifisch), sollten etwa $10^{8}$ bis $10^{10}$ Bakterien im Lebensmittelprodukt oder als Lebensmittelergänzung (Supplement) eingesetzt werden.

Die präklinische Charakterisierung von probiotischen Bakterien stellt noch immer eine wichtige Vorraussetzung dar, um die Sicherheit der Stämme, aber auch ihr mögliches Wirkungs- 
spektrum abzuschätzen. Die so erhaltenen Daten erlauben einen gezielteren Einsatz von probiotischen Mikroorganismen im gesunden, geschwächten oder, unter medizinischer Kontrolle, auch im chronisch erkrankten Menschen.

Zusammen mit einer molekularen Analyse des probiotischen Stammes basiert die Risiko-Nutzen-Abschätzung somit auf einem abgesicherten molekularen Datensatz.

\section{Schlussfolgerung}

Obgleich die Interaktionen der intestinalen Mikroflora mit dem Gesamtorganismus schon zu Zeiten L. PASTEUR's und E. Metchnikoff's antizipiert wurden, macht es erst die heutige Technologie möglich, die molekulare Basis dieser Wechselwirkungen zu verstehen. Die Entschlüsselung der bakteriellen, aber auch des humanen Genoms ermöglicht den Einblick in das genetische Potential der Mikrorganismen sowie in die daraus entstehenden funktionalen oder pathophysiologischen Konsequenzen für den Menschen.

Über die Nahrung aufgenommene probiotische Milchsäurebakterien können dazu beitragen, das Gleichgewicht der natürlichen (individuellen) Mikroflora zu erhalten oder dies bei etwaigen Störungen bis zu einem gewissen Masse zu korrigieren. Die damit verbundenen immun-modulatorischen Effekte mancher probiotischer Stämme tragen zusätzlich zur Stärkung der Immunabwehr bei.

Die meisten (probiotischen) Milchsäurebakterien haben GRAS (generally recognized as safe)-Status, da sie seit Jahrzehnten Teil unserer Nahrungskette sind. Das Plus an Gesundheit, das einige probiotische Stämme erzielen können, sollte in korrekt ausgeführten klinischen Studien dokumentiert sein.

Probiotisch angereicherte Lebensmittel oder Ernährungsergänzungsprodukte (Supplements) können auf einfache und kosteneffektive Weise dazu beitragen, das Gesundheitskapital zu erhalten oder als diätetische Ergänzung medizinische Therapien zu unterstützen.

\section{Literatur}

(1) Fuller R. History and development of probiotics. In: Fuller R, editor. London: Chapman \& Hall, 1992: 1-8.

(2) Bernet MF, Brassart D, Neeser JR, Servin AL. Lactobacillus acidophilus LA 1 binds to cultured human intestinal cell lines and inhibits cell attachment and cell invasion by enterovirulent bacteria. Gut 1994; 35(4):483-489.

(3) Isolauri E, Kirjavainen PV, Salminen S. Probiotics: a role in the treatment of intestinal infection and inflammation? Gut 2002; 50 Suppl 3:III54-III59.

(4) Isolauri E, Juntunen M, Rautanen T, Sillanaukee P, Koivula T. A human Lactobacillus strain (Lactobacillus casei sp strain GG) promotes recovery from acute diarrhea in children. Pediatrics 1991; 88(1):90-97.

(5) Schiffrin EJ, Rochat F, Link-Amster H, Aeschlimann JM, Donnet-Hughes A. Immunomodulation of human blood cells following the ingestion of lactic acid bacteria. J Dairy Sci 1995; 78:491-497.

(6) Donnet-Hughes A, Rochat F, Serrant P, Aeschlimann JM, Schiffrin EJ. Modulation of nonspecific mechanisms of defense by lactic acid bacteria: effective dose. J Dairy Sci 1999; 82(5):863-869.

(7) Mattila-Sandholm T, Blum S. Probiotics: towards demonstrating efficiency. Trends Food Science \& Technology 1999; 10:393-399.

(8) Bunout D, Barrera G, Hirsch S, Gattas V, de la Maza MP, Haschke F, Steenhout P, Klassen P, Hager C, Avendano M, Petermann M, Munoz C. Effects of a nutritional supplement on the immune response and cytokine production in free-living Chilean elderly. J Parenter Enteral Nutr 2004; 28(5):348-354.

(9) Bunout D, Hirsch S, Pia dIM, Munoz C, Haschke F, Steenhout P, Klassen P, Barrera G, Gattas V, Petermann M. Effects of prebiotics on the immune response to vaccination in the elderly. J Parenter Enteral Nutr 2002; 26(6):372-376

(10) Link-Amster H, Rochat F, Saudan KY, Mignot $\mathrm{O}$. Aeschlimann JM. Modulation of a specific humoral immune response and changes in intestinal flora mediated through fermented milk intake [published errata appear in FEMS Immunol Med Microbiol 1995 Sep; 12(1):83 and 1995 Dec;12(3-4):273]. FEMS Immunol Med Microbiol 1994; 10(1):55-63.

(11) Marteau P, Minekus M, Havenaar R, Huis i, V. Survival of lactic acid bacteria in a dynamic model of the stomach and small intestine: validation and the effects of bile. J Dairy Sci 1997; 80:1031-1037

(12) Bergogne-Berezin E. Treatment and prevention of antibiotic associated diarrhea. Int J Antimicrob Agents 2000; 16(4):521-526.

(13) Marteau PR, de Vrese M, Cellier CJ, Schrezenmeir J. Protection from gastrointestinal diseases with the use of probiotics. Am J Clin Nutr 2001; 73(2 Suppl):430S-436S.

(14) Isolauri E, Joensuu J, Suomalainen H, Luomala $M$, Vesikari T. Improved immunogenicity of oral $\mathrm{D} \times \mathrm{RRV}$ reassortant rotavirus vaccine by Lactobacillus casei GG. Vaccine 1995;(78):1597-1606.

(15) Saavedra JM, Bauman NA, Oung I, Perman JA, Yolken RH. Feeding of Bifidobacterium bifidum and Streptococcus thermophilus to infants in hospital for prevention of diarrhoea and shedding of rotavirus. Lancet 1994; 344(8929):1046-1049.

(16) Szajewska H, Mrukowicz JZ. Probiotics in prevention of antibiotic-associated diarrhea: meta-analysis. J Pediatr 2003; 142(1):85.

(17) Michetti P, Dorta G, Wiesel PH, Brassart D, Verdu E, Herranz M, Felley C, Porta N, Rou- vet $M$, Blum AL, Corthesy-Theulaz I. Effect of whey-based culture supernatant of Lactobacillus acidophilus (johnsonii) La1 on Helicobacter pylori infection in humans. Digestion 1999; 60(3):203-209.

(18) Felley CP, Corthesy-Theulaz I, Rivero JL, Sipponen $P$, Kaufmann M, Bauerfeind $P$, Wiesel $\mathrm{PH}$, Brassart D, Pfeifer A, Blum AL, Michetti P. Favourable effect of an acidified milk (LC-1) on Helicobacter pylori gastritis in man. Eur $\mathrm{J}$ Gastroenterol Hepatol 2001; 13(1):25-29.

(19) Pantoflickova D, Corthesy-Theulaz I, Dorta G, Stolte M, Isler P, Rochat F, Enslen M, Blum $A L$. Favourable effect of regular intake of fermented milk containing Lactobacillus johnsonii on Helicobacter pylori associated gastritis. Aliment Pharmacol Ther 2003;18(8):805813.

(20) Gionchetti P, Rizzello F, Venturi A, Brigidi P, Matteuzzi D, Bazzocchi G, Poggioli G, Miglioli M. Campieri M. Oral bacteriotherapy as maintenance treatment in patients with chronic pouchitis: a double-blind, placebo-controlled trial. Gastroenterology 2000; 119(2):305-309.

(21) Schultz M, Timmer A, Herfarth $\mathrm{HH}$, Sartor RB, Vanderhoof JA, Rath HC. Lactobacillus GG in inducing and maintaining remission of Crohn's disease. BMC Gastroenterol 2004; 4(1):5.

(22) Pridmore RD, Berger B, Desiere F, Vilanova D, Barretto C, Pittet AC, Zwahlen MC, Rouvet $M$, Altermann $E$, Barrangou $R$, Mollet $B$, Merœnier A, Klaenhammer T, Arigoni F, Schell MA. The genome sequence of the probiotic intestinal bacterium Lactobacillus johnsonii NCC 533. Proc Natl Acad Sci U S A 2004; 101(8):2512-2517.

(23) Schell MA, Karmirantzou M, Snel B, Vilanova D. Berger B, Pessi G, Zwahlen MC, Desiere F, Bork P, Delley M, Pridmore RD, Arigoni F. The genome sequence of Bifidobacterium longum reflects its adaptation to the human gastrointestinal tract. Proc Natl Acad Sci U S A 2002; 99(22):14422-14427.

(24) Haller D, Bode C, Hammes WP, Pfeifer AM, Schiffrin EJ, Blum S. Non-pathogenic bacteria elicit a differential cytokine response by intestinal epithelial cell/leucocyte co-cultures. Gut 2000; 47(1):79-87.

(25) Blum S, Schiffrin EJ. Intestinal microflora and homeostasis of the mucosal immune response: implications for probiotic bacteria? Curr Issues Intest Microbiol 2003; 4(2):53-60.

(26) Haller D, Serrant P, Peruisseau G, Bode C, Hammes WP, Schiffrin E, Blum S. IL-10 producing CD14low monocytes inhibit lymphocyte-dependent activation of intestinal epithelial cells by commensal bacteria. Microbiol Immunol 2002; 46(3):195-205.

(27) Blum S, Alvarez S, Haller D, Perez P, Schiffrin EJ. Intestinal microflora and the interaction with immunocompetent cells. Antonie Van Leeuwenhoek 1999; 76(1-4):199-205.

(28) Haller D, Blum S, Bode C, Hammes WP, Schiffrin EJ. Activation of human peripheral blood mononuclear cells by nonpathogenic bacteria in vitro: evidence of NK cells as primary targets. Infect Immun 2000; 68(2):752759.

(29) Haller D, Serrant P, Granato D, Schiffrin EJ Blum S. Activation of human NK cells by staphylococci and lactobacilli requires cell contact-dependent costimulation by autologous monocytes. Clin Diagn Lab Immunol 2002; 9(3):649-657.

\section{Anschrift der Autorin:}

Dr. Stephanie Blum

Nestlé Forschungszentrum

Vers-chez-les-Blanc, CH-1000 Lausanne 26

stephanie.blum-sperisen@rdls.nestle.com 